\title{
Les tribulations d'une Bible dans les médias
}

Le dossier de presse de la «Bible des écrivains »

\section{Pierre Lassave}

\section{CpenEdition}

Journals

Édition électronique

URL : http://journals.openedition.org/assr/3409

DOI : 10.4000/assr.3409

ISSN : 1777-5825

Éditeur

Éditions de l'EHESS

\section{Édition imprimée}

Date de publication : 1 mai 2006

Pagination : 9-35

ISBN : 2-7132-2092-0

ISSN : 0335-5985

\section{Référence électronique}

Pierre Lassave, "Les tribulations d'une Bible dans les médias », Archives de sciences sociales des religions [En ligne], 134 | avril - juin 2006, mis en ligne le 11 juillet 2009, consulté le 30 avril 2019. URL http://journals.openedition.org/assr/3409; DOI : 10.4000/assr.3409 


\section{Pierre Lassave}

\section{Les tribulations d'une Bible dans les médias Le dossier de presse de la "Bible des écrivains"}

Lors d'une conférence publique sur "Le fait religieux dans la presse ", un journaliste expert en religions pointait le décalage entre l'irruption sans précédent du thème religieux dans l'espace public actuel et l'incapacité structurelle de la presse à en rendre compte dans sa complexité ${ }^{1}$. Il référait ce décalage à quatre points de tensions entre les Églises et les médias : le message intégral et complexe des Églises versus l'impératif de sélection et de simplification du discours journalistique; le goût du secret propre aux autorités religieuses versus le goût de la mise en scène médiatique ; la priorité accordée à la vie collective des confessions et communautés versus le besoin de personnalisation des faits relatés; le sens du consensus ecclésial versus les catégories polémiques de l'espace politique ${ }^{2}$. Acteur de son propre discours, l'orateur ne pouvait que déplorer ces «malentendus » et appeler positivement à les réduire, notamment par une formation plus sérieuse des journalistes à la spécificité des différentes traditions religieuses. Il n'était pas dans son rôle de dire qu'il se trouvait au carrefour vivant de mondes sociaux différents mais rendus interdépendants par le contexte global de recomposition des valeurs en «modernité avancée ${ }^{3}$.

1. Henri Tincq du journal Le Monde. Conférence-débat à l'Institut Européen des Sciences des Religions, Paris, le 7 janvier 2004.

2. Points que l'on trouve initialement développés dans Henri TINCQ, "Église-médias: la double méprise ", in Pierre BrÉCHon, Jean-Paul Willaime, dirs, Médias et religions en miroir, Paris, Presses universitaires de France, 2000, p. 171-175.

3. Ce syntagme sociologique lourd de controverses désigne l'inflexion ou la radicalisation des tendances de la modernité classique telles que la rationalisation du monde, l'individualisation des comportements ou la différenciation des organisations. Divers paradigmes renouvellent actuellement cette macrosociologie: la société du risque (U. Beck), la culture réflexive (A. Giddens), la démocratisation individualiste (M. Gauchet), le système de différenciations (N. Luhmann) ou la rationalisation communicationnelle (J. Habermas) pour ne citer que ceux qui s'appuient principalement sur une observation des sociétés occidentales (cf. Yves BONNY, Sociologie du temps présent, Modernité avancée ou postmodernité?, Paris, Armand Collin, coll. «U », 2004). 
Deux ans auparavant, la presse française se mobilisait de façon spectaculaire autour d'une « Nouvelle traduction » de la Bible ${ }^{4}$. Dossier de presse moins lourd et récurrent que celui des signes religieux à l'école, mais dont le traitement intensif en France, et simultanément au Québec, en Belgique et en Suisse, constitue un intéressant terrain d'étude des formes médiatiques de reconversion culturelle d'un héritage religieux central. Plus qu'un cas de « mise en miroir » des médias et des religions, cet épisode est aussi un "événement littéraire » à part entière, ce qui ajoute à sa complexité exemplaire.

Au terme de six années d'un travail associant deux à deux, pour chacun des soixante-treize livres de la Bible catholique, une trentaine d'exégètes professionnels et une vingtaine d'écrivains de renom, Bayard-Médiaspaul lance à la rentrée littéraire 2001 un volumineux et élégant pavé de 3200 pages à prix cassé. Une Bible « à lire avec curiosité et plaisir, proche des façons d'écrire, de raconter, en français aujourd'hui $»^{5}$. Le produit s'inscrit dans une conjoncture remarquable de diversification du marché biblique de langue française à l'approche du troisième millénaire, avec la rénovation des grandes traductions de référence (Bible de Jérusalem côté catholique, Nouvelle Bible Segond côté protestant), la création de versions en français courant (Bible Parole de Vie), la réédition d'anciennes bibles célèbres (Lemaistre de Sacy, Luther), la poursuite de traductions originales qui tentent de retrouver la saveur originelle de l'hébreu (Chouraqui, Meschonnic) ${ }^{6}$. Entre les traductions qui servent le sens (Bible de Jérusalem, Traduction Ecuménique, Bible Segond) et celles qui remontent à la source (Chouraqui, Septante), la Bible Nouvelle Traduction (BNT) se situe du côté de l'expérimentation poétique (à l'instar de l'œuvre solitaire de Henri Meschonnic), segment le plus intellectuel du marché, tout en visant un public plus large (des 120000 premiers exemplaires vendus dans l'année de lancement, $15 \%$ l'ont été en grandes surfaces). L'entreprise, dont l'investissement s'est chiffré à une dizaine de millions de francs, résulte d'objectifs et de moyens multiples, sinon divergents : une visée résolument littéraire (retraduire les Écritures saintes comme tout autre texte antique en récusant autant la «belle infidèle " que le «calque archaïsant»), un appareil laïque (auteurs croyants ou non croyants, catholiques, protestants ou juifs, recrutés pour leurs compétences scientifiques ou littéraires), un statut éditorial particulier (Bayard, éditeur qui se veut indépendant mais reste lié au groupe de presse assomptionniste et s'associe pour la circonstance au catholique Médiaspaul). Le volume n'a pas pour autant obtenu l'imprimatur ecclésial à cause des bouleversements de langage qu’il introduit, mais il est recommandé par l'épiscopat à titre de traduction de

4. Éditée par le groupe Bayard en association avec Médiaspaul au Canada.

5. Publicité de l'éditeur (2001).

6. Malgré des estimations contrastées, le niveau moyen de ventes annuelles, toutes éditions confondues, se situe autour de 250000 exemplaires, ce qui n'est pas négligeable au vu de la crise contemporaine du livre. 
qualité, hors de tout usage liturgique ${ }^{7}$. Nouvel avatar de l'histoire française de la traduction biblique longtemps marquée par la fixation exclusive sur la Vulgate latine de saint Jérôme ${ }^{8}$ ? Les promoteurs de la BNT ont, en tout cas, l'ambition de doter la francophonie d'une œuvre contemporaine digne de la King James Version (1611).

Cependant, la mobilisation médiatique qui a accompagné la sortie du livre semble moins refléter cette ambition que la pluralité d'intérêts investis dans l'affaire et les effets de reclassement symbolique qu'elle produit. Par les faits qu'elle monte en épingle comme par ceux qu'elle tait, la presse joue bien son rôle de miroir plus ou moins révélateur des rapports qu'une société entretient avec ses textes fondateurs et ses croyances, que celles-ci soient religieuses, littéraires ou scientifiques. L'analyse du discours, des formes et de la dynamique même de cette campagne de presse vise ainsi à dresser un premier état des lieux des conflits et alliances qui traversent la traduction en question. Au-delà de la seule confrontation entre médias et religions, nous tenterons d'esquisser la logique sociale d'un matériau empirique pour le moins foisonnant. S'il ne s'agit pas d'une étude de réception élargie au lecteur ordinaire, les raisons et les émotions produites et transmises par les médias dessinent les contours de publics divers qui confèrent à cette retraduction biblique sa qualité d'événement culturel ${ }^{9}$.

Par «dossier de presse », nous entendons ici près d'un millier d'entrées (articles, notes, entretiens, dossiers) réparties entre le quotidien ou le magazine grand public (national ou régional) et l'organe confessionnel, catholique principalement, plus ou moins ciblé et au rayonnement variable (du quotidien

7. Modèle de casuistique, l'avis dilatoire de la Commission doctrinale des Évêques de France mérite d'être cité in extenso: "Si elle estime que cette traduction de la Bible ne peut faire l'objet d'une utilisation liturgique, la Commission doctrinale des Évêques de France reconnaît que l'appareil critique comportant introduction, notes et glossaires permet d'inscrire cette traduction dans la tradition vivante de la foi catholique. Attentive au travail engagé par les éditeurs et désireuse de le soutenir, elle a néanmoins décidé de prendre le temps nécessaire pour vérifier la réception de cette nouvelle version par les catholiques et pour apprécier sa fidélité profonde à la révélation divine. Sachant que les Écritures saintes ont toujours été l'objet d'expressions culturelles, en particulier dans la musique et les arts plastiques, la Commission doctrinale souligne l'importance de cette traduction; elle en reconnaît la portée littéraire et elle en encourage la lecture » (BNT, copyright).

8. Sur cette histoire, voir Pierre GIBERT, " Pourquoi une nouvelle traduction de la Bible ?", Esprit, mai 2002, p. 189-197; Pierre-Maurice BOGAERT, "La Bible en français ", in Robert Alter, Frank Kermode, dirs, Encyclopédie littéraire de la Bible, Paris, Bayard, 2003, p. 785 810 ; Gilles DorIval, "Modernité des traductions anciennes de la Bible ? ", Nicole GuEUNIER, «Les traductions modernes de la Bible», in Jean-Claude EsLIN, dir., La Bible, 2000 ans de lectures, Paris, Desclée de Brouwer, 2003, p. 19-47.

9. Précisons que cette étude s'inscrit dans une monographie sociologique de la BNT qui croise trois perspectives : la critique littéraire des choix de traduction opérés ; l'histoire sociale du projet et de sa réception; sa place dans les parcours individuels mobilisés. Cf. Pierre Lassave, Bible: la traduction des alliances. Enquête sur un événement littéraire, Paris, L'Harmattan, coll. " Logiques sociales ", 2005. 
La Croix, par exemple, aux multiples bulletins diocésains). Ce corpus relève, pour l'essentiel, des canaux propres au lancement d'un «best-seller » (un mois après sa sortie, l'ouvrage caracole en tête des palmarès du livre vendu) ${ }^{10}$.

\section{Dans la mêlée}

Plusieurs mois avant la sortie en librairie, divers journalistes ont été mis au fait de ce qui peut créer l'événement de la rentrée littéraire. Le projet du Nouvel Observateur de réaliser un dossier spécial sur cette traduction pour sa livraison de fin août décide l'éditeur à avancer la date de publication au 29 août au lieu du 12 septembre initialement prévu. Bien lui en a pris, car dès le 11 septembre, l'espace public se trouve totalement absorbé par l'attentat du World Trade Center. Mais cette polarisation n'a que très provisoirement nui à l'écho de l'ouvrage dans la grande presse. Si le soir du 11 septembre, un reportage de Geneviève Moll prévu au journal télévisé de 20 heures est différé sur France 2, quelques jours plus tard, la directrice de France-Culture maintient une soirée de lecture publique ${ }^{11}$. Laure Adler aurait pris cette décision après être tombée par hasard sur le passage suivant d'Ezéchiel $(1,4-5)$ : «Regardez, c'est un vent du nord qui souffle en tempête, c'est un grand nuage, c'est du feu qui jaillit entouré de lueur avec, au centre, un éclat métallique. Et dans le feu, regardez, il y a quelque chose comme des vivants ". "Il faut que cette soirée, précisera-t-elle au début de l'émission, soit un ressourcement ». L'anecdote en dit long sur l'actualité et l'universalité du patrimoine biblique aux yeux des professionnels de l'information. La mise en débat de la thèse américaine du « choc des civilisations » aurait ainsi stimulé les ventes de traductions françaises du Coran et par réplication, de la Bible (Télérama, 19/12/01). La BNT ne pouvait donc pas mieux tomber en cette période où les Écritures saintes semblent avoir paradoxalement partie liée avec la violence la plus spectaculaire ${ }^{12}$.

Dans un premier temps, de fin août à octobre 2001, les principaux journaux et magazines mettent en avant ce qu'il y a de nouveau et d'insolite dans cette

10. Ce dossier est celui de la documentation du groupe Bayard Presse sur une période allant de juin 2001 à décembre 2003 et couvrant quatre espaces nationaux (France, Canada, Belgique, Suisse). Son exhaustivité a été vérifiée et complétée par internet. Il exclut également le courrier des lecteurs, directement reçu chez Bayard dont l'indigence contraste avec l'abondance des publications. Phénomène inverse de l'accueil public de l'exposition sur l'image du Christ à la National Gallery de Londres (Seeing Salvation, 2000), où le courrier des visiteurs, nettement plus abondant que le dossier de presse, fut un matériau précieux pour la connaissance sociologique d'un autre événement significatif du recyclage culturel du christianisme. Voir Grace DAVIE, "Seeing Salvation: The Use of Text as Data in the Sociology of Religion ", in Paul AvIS, ed., Public Faith? The State of Religious Belief and Practice in Britain, Londres, SPCK 2003, p. 28-44,

11. Sources: pour France 2, Télé 7 Jours du 30 septembre 2001 ; pour France-Culture, Bulletin de l'Église d'Arras, 2 novembre 2001.

12. Régis DeBRAY, Le feu sacré, fonctions du religieux, Paris, Fayard, 2003. 
"Bible des écrivains ». La mise en scène de l'événement s'accompagne dans la presse catholique de la mise en exergue des différences entre la «Bible Bayard» et les versions de référence, notamment celle de Jérusalem (BJ). Dans un second temps, de novembre à décembre, l'événement littéraire passé, la grande presse laïque, parisienne et régionale n'en parle plus; elle n'y reviendra qu'à l'approche des cadeaux de fin d'année. Mais les divers organes du monde catholique passent alors la «Bible Bayard» au feu d'une critique généralement bienveillante et nuancée, à l'image de l'avis équivoque de la commission épiscopale que les multiples bulletins diocésains vont relayer jusqu'au printemps 2002. Une virulente campagne émanant des milieux intransigeants opposés à cette «Bible moderniste » n'est sans doute pas étrangère à la mobilisation des médias catholiques. Dans un troisième temps, ou plutôt dans une temporalité plus longue et détachée de la pression éditoriale, divers articles et dossiers sur les Livres sacrés feront retour sur cette traduction parmi d'autres et à l'occasion de la sortie de ses produits dérivés (extraits illustrés et mis en musique, formules de poche, etc.) ${ }^{13}$.

Les variations de points de vue entre les grandes presses, française, québécoise, suisse et belge, mettent en relief les formes nationales d'emprise médiatique sur les œuvres culturelles. On peut ainsi se demander si la confusion ironiquement entretenue entre les registres littéraires et religieux n'est pas, pour les journalistes parisiens, une manière de surmonter la difficulté à traiter d'un objet aussi complexe que la traduction biblique. De même, dans le monde catholique, l'agressivité et l'opiniâtreté des attaques traditionalistes semblent marquer une spécificité bien française. Pour ces minorités actives qui ne manquent pas aujourd'hui d'écoute au sein de la hiérarchie romaine, la "Bible Bayard " est présentée comme le produit typique d'un modernisme attardé et du relâchement doctrinal que combat précisément la hiérarchie romaine. Où l'histoire politico-religieuse des « deux France » et des luttes entre gallicans et ultramontains revient étrangement à la mémoire. Mais examinons de plus près les contenus.

\section{Grands chiffres, vedettes et petits mots}

De «L'odyssée de la Bible, l'événement le plus excitant de la rentrée » (Inrockuptibles) au "Best-seller pour l'éternité " (La Montagne), nombre de titres usent de superlatifs pour présenter la nouvelle traduction: «superproduction » (Libération-Livres), travail " colossal » (Le Soleil), « titanesque » (Livre d'ici), "tour de force" (Le Monde), etc. Assimiler la Bible à un roman prend dans le même temps l'allure d'un slogan. Dès août, l'Agence France Presse lance «La Bible comme un roman », titre aussitôt relayé par L’Express ( Et la Bible devint roman $»$ ). En un bel ensemble, une dizaine de journaux régionaux

13. Deux ans après son lancement, la BNT fait son apparition en livres séparés dans la collection Gallimard-Folio, gage de consécration culturelle. Et en 2005, le cycle éditorial se stabilise avec la réédition du volume complet au format de poche. 
reprennent l'antienne avec variantes : "Bayard fait de la Bible un roman " ou «Le roman de la nouvelle Bible ". Ce leitmotiv a le mérite de mêler avec humour plusieurs informations : la dimension littéraire de la Bible, le côté aventureux de cette "traduction d'écrivains ", le temps qu'il a fallu pour la réaliser. Le travail même de traduction ne manque pas à l'appel : "Les habits neufs de la Bible » (Nouvel Observateur), "La Bible avec leurs mots à eux" (Elle), "Les mots neufs de la Bible " (Le Temps), "Nouvelle traduction : sacré changement " $\mathrm{La}$ Montagne), etc. L'ironie peut alors se faire plus ou moins grinçante. Pour le moins : «La Bible en état de grâce » (Télérama), "Comment réveiller la Bible au bois dormant » (Aujourd'hui Poème) ; pour le plus : «L'Évangile selon Sainte Mode» (Figaro littéraire), après «La Bible selon Saint-Germain » (Livres Hebdo). La vindicte n'est pas absente, à la marge : «Un faux en Écritures ?» (Minute), "La Bible revue et sabotée " (Valeurs actuelles).

S’il fallait classer les principaux organes de presse nationaux selon une échelle de jugement à trois niveaux (positif, équilibré, négatif), on mettrait dans la première case : La Croix, Libération-Livres, Le Nouvel Observateur, L'Express, Le Point, Marianne, Les Inrockuptibles; dans la seconde : Le Monde, L'Humanité, Télérama, Elle, Lire, Le Magazine littéraire; et dans la troisième: Le Figaro Littéraire, France Soir, Présent, Valeurs actuelles, Minute. Ce classement sommaire confirme la réprobation des relais de l'opinion conservatrice de droite et d'extrême droite, mais il indique aussi que l'engouement pour la «Bible des écrivains » ne suit pas pour autant des contours politiques précis.

Les affiches publicitaires dans le métro parisien qui présentaient le produit de façon aussi surprenante qu'austère en dressant la liste de ses ingrédients ( « 20 écrivains/27 exégètes/73 livres/3200 pages en 1 volume »), semblent avoir servi de trame au récit journalistique. La plupart des articles d'information glosent en effet sur les « grandeurs » qui qualifient (ou disqualifient pour certains) l'événement : outre les chiffres affichés, l'œuvre de traduction s'inscrit dans un "marché porteur"; elle s'ouvre sur le troisième millénaire en rénovant un «monument polyphonique » écrit et réécrit pendant un millier d'années; elle a mobilisé 10 ou 15 millions de francs selon le journal, etc. Les chiffres élèvent le niveau d'enjeux pourtant inchiffrables, tels les déplacements de forme et de sens que cette traduction "sans attache » engage. "Cette Bible nouvelle, née de notre monde désenchanté, cette Bible démaquillée, nomade, ni juive, ni protestante, ni catholique, sans étiquette, sans Église, sans secte attachée, sans mode d'emploi, s'adresse à tous les lecteurs curieux, qu'ils soient croyants ou sceptiques » (Catherine David, Nouvel Observateur, 30/08/01). Mais " enlever le Livre Saint des mains des religieux » (Christian Makarian, L'Express, 30/08/01) tient aussi de la gageure. Non sans humour, l'exégète Jean-Jacques Lavoie, partie prenante de l'aventure, en fixe les termes : "Nous avons servi deux maîtres : l'auteur (la langue source) et le lecteur (la langue cible). Bien entendu, nul ne peut servir deux maîtres » (interview, Nouvel Observateur). 
Le travail en " binômes " (mot savant repris à satiété) suscite divers développements sur la coopération inédite entre écrivains et exégètes. Dans la presse québécoise, les interviews mettent en scène le dialogue entre virtuoses de l'écriture et gardiens du dépôt sacré, pour montrer que sa «tension " réduit l'opposition simpliste entre forme et fond. Mais dans la presse parisienne, c'est surtout l'aura médiatique des écrivains impliqués dans l'affaire qui délie les plumes. Toutes catégories de presse confondues (laïque et confessionnelle), le décompte des occurrences établit le palmarès suivant : 1) Frédéric Boyer ; 2) Florence Delay ; 3) Jean Echenoz ; 4) Jacques Roubaud ; 5) François Bon ; Jean-Luc Benoziglio ; 6) Emmanuel Carrère ; 7) Marc Sevin. Leurs divers portraits photographiques scandent une iconographie qui oscille entre art sacré et péplum hollywoodien. De l'Adam de la Chapelle Sixtine au Moïse des « Dix commandements » de Cécil B. De Mille, quand ce n'est pas John Travolta, héros du film Pulp Fiction où les tueurs à gage parlent comme Ezéchiel.

Boyer arrive largement en tête des textes comme des images. Juste écho de son rôle de «maître d'œuvre principal » ou de « chef de bande ». Son histoire se confondrait d'ailleurs avec celle de cette «nouvelle Bible » si l'on en juge par cette plaisante success story du magazine Elle (17/09/01) : "C'est l'histoire d'un type de 40 ans, brillant et séduisant. Un romancier, poète et essayiste. Un normalien bizarre qui a suivi de longues études bibliques et a aussi été professeur à la prison de la Santé. Un jour, le groupe Bayard lui demande d'entrer comme éditeur dans leur vénérable et très sérieuse maison d'édition. Frédéric Boyer, le jeune homme au front barré par une grande mèche de cheveux raides, propose alors de renouveler la lecture de la Bible » (Marie-Françoise Colombani, Elle). Le jeu médiatique fait miroiter les diverses facettes de notre « beau catholique (Libération-Livres, 6/09/01) qui se démène pour faire comprendre le sens de son entreprise. Tantôt manager (L'Expansion, 10/01) : «Responsable des éditions Bayard et grand fumeur de cigarillo » qui « a eu le culot de faire retraduire le livre le plus lu au monde ", " a organisé une véritable machine de guerre pour assurer la vente ", bref « un cas de marketing qui mériterait d'être enseigné à HEC ». Tantôt prédicateur (Le Républicain lorrain) : "Avec ses cheveux agités de courants d'air invisibles, sa longue silhouette et ses vêtements noirs, Frédéric Boyer, responsable des éditions Bayard et initiateur du projet, collerait bien à l'image du prédicateur ». Personnalisation amusante que tempère à peine telle rare notice biographique neutre et détaillée dans le quotidien La Croix ( Silhouette: Frédéric Boyer, la Bible en tête ", 30/08/01). Mais ce dernier comme les autres organes de BayardPresse ne participent pas moins à l'entreprise générale de figuration avec les nombreux portraits et entretiens de Florence Delay.

Ce sont les multiples qualités d'académicienne fille d'académicien, de romancière, de traductrice, mais aussi d'actrice et de croyante qui font de l'ex-Jeanne d'Arc de Robert Bresson une sorte d'égérie de la Bible Bayard. «Les audaces d'une immortelle " (Nouvel Observateur) enrôlée dans une traduction sulfureuse 
ne manquent pas de justifications. La Croix les présente à travers diverses rubriques qui mettent en valeur un parcours intellectuel d'exception (notices biographiques, "coups de cour » de l'immortelle, discours de réception à l'Académie), le tout illustré de portraits plus intimistes que ceux de Boyer (poses souriantes de l'académicienne devant sa bibliothèque).

Dans cette prosopopée de la traduction, Echenoz, le prix Goncourt de l'équipe, fait figure de troisième héros, moins romantique que le premier, moins flamboyant que la seconde. Le romancier «non croyant » est présenté comme un «mécano de la phrase ", travaillant jour et nuit dans la «soumission absolue » au texte biblique mis à plat par l'exégète. "Cela formait autant de rébus dont il fallait tirer une phrase qui soit non seulement fidèle, mais dont le rythme ou le souffle respecte à la fois l'original et les règles propres au français » (Notre Histoire, $\mathrm{n}^{\circ}$ 191). Par contraste avec le ton superlatif de la presse, ses interviews délivrent une leçon d'humilité et montrent à l'œuvre une réécriture biblique contemporaine aux marges de manœuvre étroitement contrôlées.

Les autres personnages en vue sont appelés en scène pour certains traits spécifiques : l'avant-garde poétique de l'après-guerre avec Roubaud ; l'écrivain athée avec Bon ; le cosmopolitisme de l'écriture avec Benoziglio ; l'audace juvénile avec Carrère ; l'initiative missionnaire avec le prêtre Sevin. Ce dernier, autre initiateur du projet de traduction, est naturellement plus présent dans la presse confessionnelle où il s'efforce de justifier l'idée d'une «Bible belle à lire et à entendre » de par ses audaces. Lorsque le support se fait moins grand public, d'autres figures d'auteurs apparaissent, tels le poète Olivier Cadiot ou le dramaturge Valère Novarina pour un lectorat féru de nouveauté littéraire.

Au jeu des chiffres et des vedettes s'ajoute celui des mots. La presse de tous bords fait fond des premières «surprises » de langage, plus ou moins heureuses pour les uns ou scandaleuses pour les autres. Relevés et griefs se recoupent nettement, illustrant de façon remarquable la fameuse "circulation circulaire de l'information ${ }^{14}$. Le relevé des perles suit deux voies : celle des expressions traditionnelles bouleversées, celle des mots clés remplacés. Dans le premier cas, les premières lignes de la Genèse sont inévitables. Passer de "Au commencement, Dieu créa le ciel et la terre. Or la terre était vide et vague, les ténèbres couvraient l'abîme et un vent de Dieu agitait la surface des eaux »(BJ) à «Premiers/Dieu crée ciel/et terre/terre vide solitude/noir au-dessus des fonds/souffle de Dieu/mouvement au-dessus des eaux » donne le coup d'envoi. La métamorphose du récit en poème, l'élision des chevilles et la recomposition lapidaire fixent pour la plupart l'enjeu littéraire mais aussi philosophique de la requalification du patrimoine religieux. La presse conservatrice stigmatise ce «big-bang » sémantique : "remaniement snob»(Figaro), «Bible hip hop », "façon rap» (France Soir). Elle

14. Pierre BouRdIEU, «L'emprise du journalisme », Actes de la recherche en sciences sociales, 101-102, 1994, p. 3-9. 
dénonce de surcroît la destruction en chaîne de l'unité du message : si le prologue de Jean, censé reprendre les termes de la Genèse, retrouve en partie sa formule traditionnelle ( Au commencement...»), le «Verbe» n'en est pas moins réduit à une simple "parole". Autre expression sous les feux de la rampe : la réponse vive de Jésus aux "Séparés " (Pharisiens) qui lui demandaient de montrer un signe du ciel (Marc 8, 12) : «Quelle engeance ! Exiger un signe ! Plutôt crever ! » Évoquée par son traducteur même, le romancier Emmanuel Carrère interviewé lors d'un journal télévisé, l'interjection aurait fait bondir dès le lendemain le chiffre de ventes de 5000 exemplaires ${ }^{15}$. La presse catholique intransigeante y verra un signe fort de profanation. Enfin le changement de formule des Béatitudes, de «Heureux les pauvres en esprit... les affligés... les doux, etc.» en "Joie de ceux qui sont à bout de souffle... des éplorés... tolérants, etc. », n'est pas passé inaperçu. Les commentaires les plus légers s'interrogent sur un éventuel clin d'œil au film À bout de souffle de Godard tandis que les plus sévères voient dans l'adjectif «tolérant » une captation de l'évangile par le "politiquement correct » dans l'air du temps. Les analyses de style correspondent mal à l'impératif journalistique du «faire simple et court ». D'où la longue litanie de mots épinglés, heureusement nouveaux pour les uns, scandaleusement déplacés pour les autres (Péché- > « égarement »; Résurrection- > « relèvement »; Esprit- > « souffle »; Foi- > « confiance »; Verbe- > « parole»; Gloire- > « rayonnement »; Satan- > " adversaire »; Arche- > " coffre "; Yahvé- > "Yhwh », etc.). Relever ces déplacements sans plus de commentaire suffit à fixer la nouveauté pour justifier la place que le journal ou le magazine lui accorde. Dans le cas des attaques intransigeantes, le lecteur non initié s'étonnera de leur caractère allusif et arbitraire. Mais dans l'ensemble, la focalisation sur les changements lexicaux s'en tient à montrer que « cette traduction frappe par sa musicalité et l'emploi d'un vocabulaire moderne » (phrase clé reprise en leitmotiv dans la presse régionale).

\section{La critique littéraire en souffrance}

Ce sont naturellement les journaux les plus intellectuels qui ont tenté de dépasser l'épiphénomène des mots pour esquisser une critique du style de la traduction. Aujourd'hui Poème, mensuel littéraire plus ou moins confidentiel, note ainsi l'émiettement prosodique d'une Genèse qui, à la différence de Meschonnic, n'a pas récupéré l'ancien phrasé massorétique; il pointe aussi la découpe néofuturiste des Psaumes ou le bonheur du haiku en quatrain qui fait revivre l'ecclésiaste (Qohélet). Mais l'analyse en reste le plus souvent au constat des genres littéraires et formes poétiques reconnues ou apparentées (Nouvel Observateur,

15. Selon Frédéric Boyer, la vingtaine de minutes télévisées réparties sur la dernière semaine du mois d'août 2001 a été décisive pour l'image et la vente. Sans journal télévisé, point de salut pour un best-seller. À noter également que dans la version poche de 2005, les éditeurs font une concession à la critique : l'exclamation de Jésus «Plutôt crever » s'atténue quelque peu en «Plutôt mourir ". 
Libération-Livres, Le Monde des livres). L'apologie du tour de force cède le pas à la litanie des difficultés du "chantier " ouvert. Le Monde des livres (7/09/01) préfère ainsi faire parler «les ouvriers dans le chantier du Livre » plutôt que de développer la critique à chaud d'une opération aussi complexe et chargée d'enjeux philosophiques et littéraires. Et nos ouvriers d'exprimer le «choc de l'expérience » de traduction biblique et toutes ses limites: les «spécialistes de l'usure du langage » (Florence Delay) ont tenté « d'entourer au mieux le mot à mot » exégétique (Olivier Cadiot), bien conscients de ne pouvoir épuiser le sens d'un texte énigmatique qui parle « de la possibilité, du désir, de la difficulté de croire » (Pierre Alferi). Le dépouillement du style fait corps avec une entreprise qui tient finalement plus de Lilliput que de Prométhée. Libération-Livres (6/09/01) parle de "Bible à l'os »: «Le présent chasse souvent le passé. Les dialogues sont plus directs; les phrases plus courtes que dans les éditions classiques. Des vers secs, libres, rapides, sont jetés comme des pierres sur la page et dans notre imaginaire habitué aux longues périodes et à l'apaisement de la langue française classique " (Philippe Lançon). Le Figaro littéraire (13/09/01) parle de "Bible gadget " à "l'écriture blanche, frigide, anorexique, qui fait le bonheur des éditions de Minuit et de POL» (Sébastien Lapaque).

Entre ces avis juxtaposés s'esquisse un débat plus ou moins larvé sur les liens entre la forme et le fond. Dans une tribune libre de Sud-Ouest Dimanche (9/09/01), Dominique Barrios, traductrice, membre des équipes de la Traduction œecuménique (TOB) et de la BJ, directrice littéraire aux éditions du Cerf (concurrent direct de Bayard), estime ainsi que «si le traducteur de la Bible ne respecte pas indissociablement le sens et le style, il y a fort à parier qu'il passera à côté du texte ». Il convient alors d'éviter que le style propre d'un traducteur, surtout lorsqu'il est écrivain, déteigne sur celui du texte à traduire. C'est ce qu'elle semble indirectement reprocher à la mise en scène publicitaire de tel romancier connu : «On ne traduit pas une œuvre littéraire, quelle qu’elle soit, en négligeant le fait que c'est une œuvre littéraire, que sa forme fait partie de sa signification même. À l'oublier, on risquerait de prendre Josué 10, 12-13 pour une chronique historique ou un compte-rendu météorologique, et non pour un morceau de poésie épique, au lyrisme comparable à notre Chanson de Roland». On devine qu’à travers Echenoz, non explicitement nommé, c'est la séparation entre écrivain et exégète qui est visée. "Démarche schizophrénique ", dit la même critique, citée dans le magazine Lire du même mois. Grief qui rejoint celui de l'inculture historique et linguistique des traducteurs repris par d'autres, tels Le Figaro littéraire qui glose sur les «écrivains ignorants» ou «laborantins» ou Meschonnic qui dénonce la supercherie d'une adaptation masquée ${ }^{16}$. Autre point de litige soulevé

16. Le linguiste, poète et traducteur dénonce un «faux en traduction » en invoquant le code de déontologie du traducteur qui exige la double maitrise de la langue de départ et d'arrivée (Henri Meschonnic, Un coup de Bible dans la philosophie, Paris, Bayard, 2004). " L'intelligence partagée de la traduction " prônée par Jacques Roubaud, son adversaire littéraire, reste 
par la directrice au Cerf, celui de l'hétérogénéité volontaire des traductions de la «Bible Bayard » qui ferait peu de cas de l'unité profonde des Écritures saintes. «Cette unité n'est pas seulement dans la parole de Dieu qui, pour les croyants, s'exprime à travers tous les livres. Elle est dans leur composition même. Il y a, entre ces livres, un incessant jeu de miroirs et d'échos. La Bible n'est pas la réunion fortuite de textes indépendants; elle est un savant tissage de fils volontairement entremêlés ». L'introduction de la BNT (F. Boyer) affirme pourtant nettement l'unité historique de la Bible, ne serait-ce qu'à travers le respect du canon catholique ou de certaines consignes minimales d'écriture ici données aux traducteurs (le tétragramme Yhwh).

Au-delà du relevé vétilleux des défauts de concordance lexicale d'un livre à l'autre (examen surdéterminé par une tradition qui a gommé au fil du temps les disparités linguistiques), la presse française éclaire peu son lecteur sur cette dialectique entre unité et diversité des Écritures. Tandis que Le Figaro littéraire stigmatise la «Babel stylistique » de la «Bible au goût du jour », L'Humanité (18/10/01) met en valeur, à l'inverse, les « contradictions internes aux Écritures elles-mêmes » que révèlerait une authentique traduction littéraire, critique et « désacralisante». Le journaliste du quotidien communiste (Arnaud Spire) cite ainsi le nihilisme foncier de l'Ecclésiaste ou de Job (par contraste avec l'optimisme évangélique, faut-il comprendre). Mais une fois de plus cette question de fond n'est pas développée.

La confusion des registres religieux et littéraires semble d'autant plus prégnante en France qu'elle offre ses jeux de langage, ses clins d'œil ironiques, à un journalisme en mal d'audience quand il aborde des sujets fort difficiles, objets de surcroît de longues controverses théologiques vouées à l'épuisement séculier. Cette idée s'est confirmée à la lecture de la presse francophone non française, où quelques articles tentent d'éclairer le public sur les enjeux contemporains de la traduction biblique. Plus qu'en France, les journaux québécois mettent ainsi l'accent sur le caractère littéraire des Écritures saintes et sur la dimension scientifique de leur nouvelle traduction. «Avec la Bible, affirme d'emblée Jacques Folch-Ribas dans le quotidien La Presse (9/09/01), l'un des principaux problèmes est qu'elle fut écrite et traduite dans le but de faire croire ". Il était temps que le lecteur contemporain redécouvre la littérature biblique comme le permet désormais l'exploit de la nouvelle traduction, conclut l'auteur. Dans un dossier spécial sur cette « Nouvelle Bible » (8-9/09/01), le quotidien Le Devoir (d'origine catholique) montre moins d'états d'âme que la presse française confrontée à la question de la sacralité du texte. "Il a été remis en mémoire que le livre des livres était, pour tout lecteur, une succession de textes possibles, où l'aléatoire de la phrase lue avait été déterminé dans les siècles passés soit par un copiste,

pour lui un leurre. À noter que Bayard n'a pas craint de publier le brûlot de Meschonnic. Magnanimité éditoriale qui veut sans doute prouver la volonté d'ouvrir un débat. 
soit par un savant convaincu ", note Normand Thériault en introduction au dossier. La nouvelle traduction fait pour lui œuvre de liberté : «La Bible a été retirée aux docteurs de la Loi et remise entre les mains de qui sait écrire, le texte étant toutefois authentifié par des spécialistes qui ont connaissance et science d'un texte premier, dans la langue originale de sa rédaction ". La distinction entre le statut historique du texte biblique et son usage sacralisant semble avoir plus de chances d'être comprise ici. D'où la mise en exergue des propos de Boyer, maintes fois répétés à cet égard: "Les textes de la Bible ne sont sacrés qu'en fonction de leur utilisation et de leur inscription dans la tradition de foi d'une communauté croyante. Sinon, on ne peut pas parler de langue ou de texte sacrés. La Bible est d'abord et avant tout humaine ».

Position de discernement qui fait encore scandale dans certaines chapelles françaises, mais que la presse belge, catholique comprise, admet pourtant comme un fait acquis. Les articles et entretiens du quotidien La Libre Belgique (13-14/ 10/01) mettent ainsi l'accent sur le travail même de traduction biblique à travers l'histoire pour justifier les libertés que la «Bible littéraire et contemporaine » prend avec le langage de la tradition. L'infatigable Boyer est à nouveau sollicité : «La Bible a toujours été traduite en fonction de la langue de réception. Comment a-t-on traduit à l'époque grecque ? En faisant des sauts culturels en s'inspirant d'Homère. Et saint Jérôme a traduit dans la langue qui sera la grande langue médiévale. " Il reçoit l'appui de Jean L’Hour, l'un des plus actifs exégètes de l'équipe, qui montre, à partir de sa propre expérience de missionnaire en Asie, qu’il y a au moins deux manières de traduire la Bible selon que le texte est destiné à la croyance religieuse ou au lecteur universel : " Je me suis rendu compte, par exemple, que des termes aussi courants que péché, gloire ou résurrection ne sont pas du tout compris à l'extérieur du sérail ». Il s'agit donc pour lui « de rendre à l'humanité quelque chose qui appartient à son patrimoine et qui n'est pas la propriété exclusive des croyants ».

Le rôle joué par les exégètes dans la nouvelle traduction est à cet égard plus présent dans la presse francophone que dans la presse française. Sans doute est-ce dû pour le Québec au fort contingent de biblistes engagés dans l'entreprise et plus généralement à l'effet de vedettariat littéraire propre à la France. Mais dans l'ensemble, quel que soit le pays, les articles, les entretiens et les reportages hésitent à entrer dans l'atelier des traducteurs. Le syndrome d'une critique qui s'essouffle au-delà des changements apparents de vocabulaire s'avère général. Seule l'information sur la philosophie générale de la traduction peut être moins confuse ou plus développée ici que là. Dans Le Devoir (13/09/01), par exemple, Caroline Montpetit lance une piste de réflexion sur la dimension «existentielle d'une traduction qui s'adresse plus directement aux individus avec leurs mots d'aujourd'hui. Elle s'appuie sur les premières discussions entre connaisseurs, par exemple autour de ce changement de vocabulaire dans l'épisode des Noces de Cana où le «Jésus manifesta sa gloire et ses disciples crurent en lui » devient : «il s'est 
montré dans tout son éclat et ses disciples lui ont fait confiance ». Pour l'abbé Guillemette, responsable du Centre catholique biblique à Montréal, ces nouveaux mots sont susceptibles de toucher "les gens qui sont en recherche existentielle ou qui s'interrogent à ce sujet ».

La question de la réception n'ira pas au-delà de quelques allusions parisiennes et plus ou moins désobligeantes à la cible «branchée » de l'ouvrage ( "La Bible selon Saint-Germain »). À peine quelques articles suggèrent-ils que le travail de traduction n'a en tout cas pas laissé ses " ouvriers" « indemnes ». "Traduction ou pas, les résonances pour chacun ont été fortes. C'est pendant ce travail que Carrère a écrit L'Adversaire (qui est le nom donné dans cette Bible à Satan), autour de l'affaire Roman. Benoziglio publie cette rentrée, "pour la première (et dernière !) fois de ma vie, un bouquin censé se passer il y a ... trois mille ans. Coïncidence?" Plus intime, l'écho de la traduction pour Florence Delay: "ce qui a été modifié en moi ? L'inconnu. L'inconnu est devenu encore plus immense." Quant à François Bon, il semble avoir pris goût à cette besogne : "forcément, cela aura un impact". Lequel, il est trop tôt pour savoir. L'envie de continuer dans son coin, sur Eschyle ou Shakespeare, peut-être. » (Belinda Cannone, Marianne, 3-9/09/01). Avec le temps, le reportage va peut-être céder la place à l'analyse et le magazine s'effacer devant la revue savante. Mais dans cet intervalle, il faut faire une place à part à la presse confessionnelle. Ses quotidiens, revues et bulletins, imprègnent en effet la critique de dimensions doctrinales et exégétiques que la presse profane ne pouvait qu'effleurer.

\section{L'accueil divers dans les chapelles}

Éditée par un grand groupe de presse, la «Bible Bayard " a largement bénéficié de la promotion faite par le quotidien La Croix et les magazines de la maison (Panorama, Pèlerin, Vermeil, Phosphore, les Dossiers de l'actualité, Écritures, etc.). D'août à novembre 2001, le quotidien catholique a publié plusieurs unes et dossiers sur l'actualité de la Bible et de sa traduction. Le premier dossier ( Quoi de neuf ? La Bible! », 30/08/01) développe plutôt les dimensions culturelles des Écritures saintes, l'histoire de leurs liens avec la littérature, mais aussi le théâtre, le cinéma. Le suivant immédiat ("Redécouvrons la Bible ", 1-2/09/01) interpelle le lecteur confessant : "Entendons-nous encore le texte biblique dans toute sa nouveauté, dans toute sa force, sous des formes auxquelles nous nous sommes habitués, peut-être jusqu'à la routine?" (Jean-François Bouthors). La «Bible Bayard» vise non seulement à « rendre la Bible à la grande littérature » mais aussi et surtout à en "réveiller la lecture » chez les croyants. Ses diverses qualités sont exhibées au fil d'articles pédagogiques outillés de références bibliographiques : traduction en binômes ( Exercice d'humilité réciproque »), renouvellement lexical, polyphonie revendiquée, encouragement épiscopal. De grands témoins se penchent sur son berceau: Mgr Pierre d'Ornellas («Cette soif 
d'écouter la parole de Dieu a son secret »), Marcel Bozonnet, administrateur de la Comédie-Française ( "Un texte qui s’affirme comme une parole qu'on a envie de lire à haute voix »), et last but not least, Florence Delay ("En suivant la voix de Saint Jean »). Les magazines du groupe renforcent les mois suivants la défense et illustration de cette "incroyable aventure " de traduction (Pèlerin, 7/09/01) au fil d'interviews imagées et vivantes (Hugues Cousin, Florence Delay). Mais les organes des groupes catholiques concurrents (ex-Malesherbes-PVC, ex-AmpèreMédia-Participations, Le Cerf) ne tardent pas à faire entendre leur voix.

Il n'y a pas que la «Bible Bayard » qui fait événement, rappelle d'abord JeanMaurice de Montrémy dans Notre Histoire, n 191 («La guerre des Bibles ») : la sortie de la traduction des Psaumes par Meschonnic (Gloires) chez Desclée de Brouwer, la traduction du Pentateuque à partir de la Septante et le lancement de la revue Biblia au Cerf sont à prendre en compte pour cette rentrée littéraire décidément fort biblique. Ces initiatives posent une double question : 1. «Un texte d'écrivains sera-t-il plus fort qu'un texte établi par les spécialistes rompus à l'exégèse ? ; 2. Ne faut-il pas rendre "le dire plus que le dit », comme s’y attelle Meschonnic ? Ce ne sont là que quelques points de débat à peine évoqués et qui n'auront que peu d'échos par la suite. Dans ce magazine, comme dans un autre du même groupe, La Vie, les questions posées à «La Bible en habits neufs ", passent surtout par celles des entretiens avec les écrivains non croyants, «irradiés" par la réécriture du texte biblique (Echenoz, Carrère, Benoziglio). Un premier retour aux questions principielles sur le statut du texte est le fait de l'hebdomadaire protestant Réforme (20-26/09/01). Si l'organe se réjouit d'une entreprise «missionnaire » qui brise la « dichotomie bien française entre culture religieuse et culture profane » (Rémy Hebding), il émet quelques réserves sur ses reconstructions narratives ou recompositions poétiques qui lui ôtent une part de son caractère sacré (Joël Schmidt). Mais l'objection reste allusive.

Moins impressionnistes sont en revanche les tirs de barrage de la mouvance intransigeante ou traditionaliste. Dans L'Homme nouveau (16/09/01), Philippe Maxence dresse un réquisitoire sans appel ("Rendez-nous la Bible!») : débauche de moyens ("Bayard sans peur et sans reproche») ; profanation des Écritures (cf. péché/égarement, résurrection/éveil, Verbe/parole) à l'encontre du concile de Vatican II ; atteinte à l'identité catholique par introduction de traducteurs juifs, protestants et non croyants. Mensuel proche de Présent et de l'extrême droite, La Nef n'est pas en reste. Dans sa livraison d'octobre, Bruno Nougayrède ( "Une bible dans le vent ») reprend les mêmes chefs d'accusation : opération mercantile, ambivalence épiscopale, "idiome de cour de récréation » et style "petit nègre ", profanation du texte sacré. Mais la palme de la critique intransigeante revient sans doute à l'hebdomadaire Famille chrétienne, au rayon de diffusion plus étendu dans l'Église catholique ${ }^{17}$. Dans la dernière livraison d'octobre, Philippe

17. Lié au groupe Média-Participations (ex-Ampère), l'hebdomadaire Famille chrétienne tourne autour de 60000 exemplaires. 
Oswald ("La Bible version tendance ") fustige le sabordement des principes conciliaires (Dei Verbum invoqué) : absence d'attention à "l'unité et au contenu de toute l'Écriture », déni de la « Tradition vivante de l'Église », oubli de « l'analogie de la foi " (cohésion des vérités de la foi entre elles). La réécriture de la Bible par des écrivains et des non-catholiques, conclut l'auteur, est « intrinsèquement rebelle » à la vénération de l'Écriture sainte par l'Église. On retrouve les mêmes pièces à conviction ( «Plutôt crever!", "réveillé », etc.) d'un procès qui s'en tient au vocabulaire et prend strictement à la lettre les textes dogmatiques de l'Église comme pour mieux éviter d'en aborder l'esprit. Les adversaires d'hier de Vatican II, sont rapidement devenus ses plus vétilleux gardiens, résurgences ultramontaines obligent.

Mais tel acte d'accusation, aussi peu argumenté et aussi marginal soit-il dans l'espace idéologique national, ne pouvait cependant rester sans réponse. Dans une troisième série de dossiers spéciaux de novembre ( La Bible, un livre pour vivre »), La Croix rétablit la dialectique conciliaire dévoyée par les interprétations littéralistes ou fondamentalistes. "Il est courant de définir la Bible comme Parole de Dieu. Mais comment faut-il l'entendre ? Le christianisme y discerne une inspiration divine, mais il n'est pas une "religion du Livre" [...]. Le texte biblique n'a pas un caractère "sacré", au sens d'une instance radicalement extérieure aux réalités humaines [...]. Il y aura donc toujours un écart entre la Bible, avec sa part de contingence, et la Parole de Dieu, totalement transcendante " (Michel Kubler). Inutile de préciser que cet «écart» constitue le lieu, le champ ou la marge de manœuvre de la nouvelle traduction en cause. Fort de ce rappel doctrinal, La Croix ouvre les fenêtres sur la lecture «vivante» de la Bible dans divers milieux sociaux et confessionnels (protestant, juif). Une photo montre ainsi un prêtre éthiopien enturbanné lisant la Bible à la bougie ; légende : "Chaque époque et chaque lecteur, mis en face des mêmes textes, pose à nouveau la question du sens ».

Mais la sortie en librairie d'une nouvelle édition de la Bible de Jérusalem, version grand public et avec « clefs de lecture » étroitement inspirées des Pères de l'Église, relance « la guerre des Bibles ». Dans une interview accordée à Famille chrétienne (1-7/12/01), Nicolas-Jean Sed, dominicain et directeur du Cerf (coéditeur de la BJ avec Fleurus-Mame) fait la promotion de cette nouvelle édition qui associe étroitement travail exégétique, redécouverte de la tradition et renouveau liturgique. Interrogé sur la «Bible Bayard", il répond d'abord par une exclamation de mépris : "Oh vous savez la Bible de Bayard... (silence) ». Puis se ravise pour délivrer un jugement sans appel : "J'aime beaucoup les transpositions littéraires de la Bible [...], mais il est exclu qu'on puisse remplacer le mot "Résurrection" par les mots "éveil" ou "relèvement" [...]. Du moment que ces mots sont habités par la prière et par la prédication, ils deviennent non seulement indispensables mais irréversibles. Je ne veux pas de l'émancipation de l'abbé Sevin. [...]. Et je sais ce qu'il en coûte de "sortir de ce parler chrétien" ». Le 
directeur fait ici allusion à la catastrophe de l'émancipation communiste qu'il a côtoyée de près par sa famille issue d'Europe centrale.

Sans qu'il s'agisse d'une réponse directe à ces attaques, La Croix (14/12/01) revient sur le succès commercial de la "Nouvelle traduction » (140000 exemplaires déjà vendus dans le monde) et ouvre un forum pour débattre des questions qu'elle soulève «parmi les fidèles et certains pasteurs »: "Le langage de la "Bible Bayard" serait-il éloigné des expressions reçues, notamment à travers les lectures de la messe ? Cette Bible, non destinée à la liturgie, peut-elle être utilisée dans la vie ecclésiale, notamment en catéchèse ? Comment interpréter le fait que, tout en voyant sa lecture encouragée par l'épiscopat, elle n'ait pas encore reçu l'imprimatur?» (M. Kubler). Divers témoins donnent alors leur avis, plus ou moins positif. «Loin d'ergoter sans fin sur tel ou tel mot, les lecteurs de la "Nouvelle traduction" veulent se nourrir de la beauté d'un texte, afin d'ouvrir leur cour à la vivante parole de Dieu », affirme ainsi Joseph Stricher, directeur du Service biblique catholique Évangile et Vie. «L'enjeu de cette traduction me paraît moins pastoral que missionnaire. Elle peut être un chemin spirituel pour ceux qui ne se reconnaissent pas dans la proposition chrétienne », estime Mgr Georges Gilson, archevêque de Sens-Auxerre. Si cette traduction ne semble pas pouvoir servir la catéchèse (Anne Mayol, directrice de la catéchèse pour le diocèse de Paris), elle fait en tout cas parler d'elle et ravive ainsi l'intérêt de sa lecture (Annie Wellens, libraire).

À travers quelques petites phrases glanées par notre libraire, s'esquisse une première image empirique de la diversité des publics de la BNT : «Enfin, je vais pouvoir offrir la Bible sans qu'on me soupçonne de prosélytisme " dit bien l'attrait de sa relative neutralité confessionnelle. "Les plus heureux sont des littéraires, souvent lecteurs des auteurs concernés par la nouvelle Bible. Ils sont intéressés par la culture juive ou chrétienne, mais ne se définissent pas comme croyants ", précise la libraire. Pour les chrétiens affirmés, c'est une traduction à prendre en compte; pour les responsables ecclésiaux, c'est du travail en plus ; pour un prêtre perplexe, "elle ne vieillira pas »; pour un laï qui fait du mauvais esprit : " Achetez cette Bible, plutôt crever!". "Promouvoir dans cette réécriture de la Bible un texte "déconfessionnalisé" reviendrait à faire l'éloge d'un vin sans alcool ", estime pour sa part la libraire.

De la base au sommet, ce qui reste de forces lectrices dans l'Église est manifestement partagé à l'égard de la «Bible Bayard ». Les attaques émanant des milieux traditionalistes ne doivent pas cependant cacher le ton mesuré qui domine les publications catholiques. La plupart des analyses reconnaissent le sérieux de l'appareil exégétique et la créativité de la réécriture, mais font état des limites théologiques imposées tant par l'hétérogénéité des traductions que par les innovations lexicales (Notre Histoire, Actualités des religions, Le Nouvel Informateur Catholique). Partout la Bible de Jérusalem reste la référence indépassable. La TOB arrive en seconde position. Quelques timides voix au Québec (Relations) 
et en Belgique (Choisir) s'interrogent sur l'accessibilité sociale d'une nouvelle «traduction littéraire » qui joue sur une grande diversité de styles. Tendance à l'élitisme culturel qui risque de réduire l'ambition missionnaire aux publics les plus lettrés. Mais l'objection, comme beaucoup d'autres, ne fera pas débat.

Au cours de l'année 2002, la pression médiatique retombe. Quelques prises de position épisodiques reviendront sur les qualités et les défauts de ce qui restera pour les uns la «Bible Bayard» et pour les autres la «Bible des écrivains» ( $\mathrm{La}$ Voix protestante, Chrétiens en marche). Seule la mouvance traditionaliste rappelle périodiquement son indignation devant ce nouvel avatar du « modernisme " $\mathrm{La}$ Net, 02/2003). Mais de l'automne 2001 au printemps 2002, une multitude de bulletins diocésains présentent la «Nouvelle traduction ». Ils se rangent pour la plupart à l'avis nuancé de la Commission épiscopale. Catéchistes, prêtres et exégètes convergent sur le fait que cette traduction ne remplace pas la $\mathrm{BJ}$ et encore moins la Bible liturgique en voie d'achèvement. Ils apprécient cependant en elle une œuvre littéraire qui renouvelle l'écoute de la parole divine. «Bible à savourer » plus que les autres, la métaphore gustative nourrit l'idée de lectio divina à la manière hédoniste ou charismatique d'aujourd'hui. Revenant sur la question de sa lisibilité sociale, Mgr Émile Marcus, archevêque de Toulouse (Bulletin diocésain "Foi et Vie », 10/02/02) écrit : «Elle pique ma curiosité mais je ne trouve pas qu'elle soit plus accessible que d'autres auxquelles il faudrait la préférer à cause de la distance qu'elle aurait prise par rapport à leur "langue érudite et leur français académique” ». Babel pèse toujours sur l'universalité du message chrétien quelles que soient les pentecôtes de la traduction.

\section{Lignes de tensions}

Plus que toute autre traduction française, la «Bible des écrivains » a tiré les conséquences littéraires de la relativité historique des Écritures. Non seulement elle renouvelle le vocabulaire de la Vulgate, mais aussi, suivant les livres, elle réanime la mise en intrigue antique (livres historiques), recrée une prosodie (livres poétiques) ou met en valeur la rhétorique argumentative (épîtres pauliniennes). Entre la re-versification créative, la dynamisation narrative et la clarification discursive, les lecteurs et les référents varient sinon s'opposent : lettré féru d'innovation, public plus large amateur de récits merveilleux, savant ou théologien en quête de la plus fidèle version, etc. Les gages donnés au canon catholique n'empêchent pas la mise en valeur de la diversité des sources et des interprétations (comme l'attestent les glossaires et les notes) ni la recherche de la « signifiance » hors de toute visée liturgique ${ }^{18}$.

18. Par signifiance, on peut entendre tout ce qui, dans l'énonciation, va au-delà du simple énoncé. Meschonnic l'a théorisée dans Poétique du traduire, Lagrasse, Verdier, 1999. 
L'animation du dossier de presse est à la mesure d'une entreprise qui révèle au grand jour les contradictions propres à la transmission contemporaine d'un héritage pour le moins indissociablement poétique, mythologique et métaphysique. L'aspect superficiel de la couverture médiatique ne renvoie pas seulement aux contraintes naturelles de la presse (faire simple et court pour attirer le maximum de lecteurs). Il témoigne aussi de l'éclatement de l'héritage biblique entre livre multiconfessionnel et " grand code de l'art » dans un contexte global d' " exculturation » du christianisme ${ }^{19}$. La difficulté du journalisme français à dépasser le seul niveau de la chasse aux mots nouveaux, apparemment moins nette dans les autres pays francophones, pourrait être également mise au compte des embarras d'une laïcité dominante qui n'aurait pas complètement réglé son complexe judéochrétien. Dans sa facture comme dans ses intentions affichées, le produit éditorial en question est imprégné de ces tensions. D’un côté, la célébration de la littérature (écrivains et mise en page créatifs) et de la science (biblistes et glossaires philologiques) au détriment de la théologie, et de l'autre, l'appel à l'autorité ecclésiale (imprimatur). D'un côté, l'invocation de la dimension historique du texte (au risque d'oublier les croyants), et de l'autre, la justification chrétienne de la traduction laïque. Notre corpus de presse s'est bien fait l'écho des conflits entre courants catholiques derrière lesquels se profilent l'incapacité des organes officiels de l'Église à statuer clairement sur le bien-fondé de la traduction ainsi que des stratégies commerciales et cléricales en sous-main. Mais il ne laisse entrevoir qu’à la marge les tensions propres à l'espace public au sens large.

Deux ans après la publication, force est de constater que le commentaire savant tarde à prendre le relais des journaux et magazines. Ce sont les experts catholiques qui se sont le plus exprimés; dans une palette de revues allant de Képhas (pour la plus confidentielle et la plus traditionaliste) à Esprit (pour la plus publique et la plus moderniste) en passant par des dossiers analytiques dans divers organes confessionnels (Croire aujourd'hui, Esprit et Vie, Foi et vie, Revue théologique de Louvain). Seule Vacarme, revue de critique culturelle liée au mouvement Act up de Paris, déroge à la règle ${ }^{20}$. Dès septembre 2001, cette revue parisienne livre en effet un important dossier d'enquête sur la BNT ( $T$ Traduire la Bible/Work in progress ») avec analyse historique des traductions françaises, comparaisons systématiques entre versions concurrentes, interviews des traducteurs, mise en contexte théorique. Cet hommage surprenant d'un groupe alternatif en faveur d'une grande entreprise d'édition catholique s'explique par les liens

19. Voir sur la sortie du catholicisme du tableau des valeurs dominantes de la France contemporaine, Danièle Hervieu-LÉger, Catholicisme, la fin d'un monde, Paris, Bayard, coll. "Questions en débat », 2003.

20. Créée en 1997, la revue Vacarme se veut un lieu de réflexion sur le mouvement social et culturel, notamment le combat des malades du sida, des chômeurs, des sans-papiers. Ses jeunes animateurs se veulent les héritiers des mouvements féministes, antipsychiatriques et d'intervention dans les prisons (Foucault) des années 1960-1970. 
de combat culturel entre la rédaction et certains écrivains aux positions d'avantgarde enrôlés dans la BNT (Alferi et Cadiot, animateurs de la feue Revue de Littérature Générale chez POL). Vacarme met l'accent sur les questions littéraires que soulève cette "expérience créative inédite sur le texte fondateur de la culture occidentale " : passage du "sens à sens " (préconisé par saint Jérôme à l'encontre du littéralisme) au "langue à langue » («Le secret peut passer d'une langue à l'autre sans traverser la conscience ») ; tension entre l'oral et l'écrit ( Il fallait que ça parle et que ce soit écrit »); maîtrise de l'écart culturel entre les sources et les cibles ( Donner l'opaque sans donner l'opacité, c'est la face claire de l'opaque, de l'ombre $»$ ). Manifestement, Vacarme légitime l'opération Bayard dans le microcosme littéraire et fait ainsi pendant à un numéro de la revue L'Infini, dirigée par l'influent Philippe Sollers, qui, au même moment, rend un hommage exclusif à l'œuvre de traduction biblique de Meschonnic (sans la moindre citation de la BNT) ${ }^{21}$.

Plutôt que de s'appesantir sur les multiples expertises confessionnelles, nous ne retiendrons d'elles qu'une critique appuyée qui rejoint celle qui, dans l'année 2002, nourrit la controverse entre Alain Finkielkraut et Frédéric Boyer, par essais et France-Culture interposés. Ainsi dans la très conservatrice revue Képhas, organe de critique doctrinale animé par des membres de la Fraternité Saint Pierre ralliés à Jean-Paul II, Olivier-Thomas Venard s'interroge-t-il sur les présupposés philosophiques et littéraires de la traduction en question ${ }^{22}$. Il oppose d'abord la «beauté toute simple» des phrases de Marie-Andrée Lamontagne (Matthieu) à la démagogie populiste du fameux «Plutôt crever!" d'Emmanuel Carrère (Marc) pour montrer que la traduction peut être socialement accessible sans pour autant parler en argot. Mais si Lamontagne cède parfois à la « langue de bois laïque et gratuite ( (cf. «Joie des tolérants...»), c'est parce que l'idéologie parle dans une traduction dont le lecteur implicite se présente plus en amateur de littérature dans l'air du temps qu'en pèlerin de la foi intemporelle. Tout en louant le bel effort de présentation textuelle avec ses variations typographiques, Venard pointe dans l'appareil critique quelque déséquilibre entre le dernier cri de la théorie littéraire (génétique et intertextualité) et l'obsolescence de certaines positions exégétiques (fausse opposition entre Jésus historique et Christ de la foi). Si la rencontre entre l'hébreu biblique et le laconisme de la poésie contemporaine s'avère ici une réussite, le critique fait craindre le procédé et montre une certaine défiance des écrivains traducteurs à l'égard du "versant prolixe » du récit. Il y voit un effet indirect de la «culture du soupçon envers la parole humaine ». Mais la déficience essentielle provient, selon l'auteur, du " parti pris contre les mots de la tribu judéo-chrétienne » (âme, résurrection, Verbe, etc.). D’où « l'excellent

21. "Coup de Bible ", L'Infini, $\mathrm{n}^{\circ}$ 76, automne 2001.

22. Olivier-Thomas Venard, "La culture de la Bible Bayard ", Képhas, 1/2002. L'auteur est dominicain, agrégé de lettres modernes, ancien élève de l'École normale supérieure de FontenaySaint-Cloud, et enseignant à l'École biblique et archéologique française de Jérusalem. 
glossaire final» qui « ressemble un peu à de l'autojustification ». Le critique s'appuie alors sur Northrop Frye pour stigmatiser la «culture immanentiste » d'une traduction qui dénierait l'efficace symbolique de la parole, pourtant capable de susciter l'être au-delà de sa seule nomination ${ }^{23}$. D'où le contresens qui guette la traduction du très théologique "Ceci est mon corps " par le trop allégorique «Ce pain est mon corps». Malgré la reprise heureuse du terme Alliance (Nouvelle), la non-concordance des termes entre l'Ancien et le Nouveau Testament alourdit le déficit théologique. L'insistance sur la diversité sémantique des livres cache mal ainsi le déni d'unité christologique de l'ensemble. S'élevant en généralité, le critique voit finalement dans la «Bible Bayard» le symptôme de la «démission culturelle de l'Église de France ». Ainsi, la dialectique traductrice entre exégète et écrivain ne ferait que reproduire une fausse dualité entre science (principe de réalité) et littérature (principe de plaisir) tout en oblitérant le sens ultime (principe de foi). «Le raffinement littéraire d'une part, et l'indigence doctrinale de l'autre, participent finalement moins d'un rejet de la religion que d'un déplacement (peut-être inconscient) du religieux vers la littérature ». Face à ce glissement fatal de valeurs, le dominicain en appelle, "sous l'invocation de saint Jérôme », à une Bible contemporaine à la fois savante, populaire et chrétienne. Appel utopique ou d'un autre temps, serait-on tenté de dire au vu de la tendance, apparemment inéluctable, à la pluralité des traductions bibliques ${ }^{24}$.

Mais le philosophe Alain Finkielkraut fait indirectement écho à la critique du dominicain. Dans son essai L'Imparfait du présent, il consacre en effet une de ses «pièces brèves » à « une nouvelle traduction française de la Bible » en accusant celle-ci de dilapider l'héritage : il ne restait plus de lui que « deux ou trois formules et quelques proverbes passés dans la langue commune ", cette nouvelle traduction l'anéantirait avec son langage de « micro-trottoir ${ }^{25}$. Le mot «péché : symptôme de la fragilité et de la faillibilité humaine, témoignage de notre incapacité à nous délivrer nous-mêmes du mal » se serait dévoyé en «égarement : excès romantique, oubli de soi, moment de folie, vertigineuse parenthèse ». $\mathrm{La}$ « foi : accueil de la hauteur, reconnaissance de l'absolu, abrupte dissymétrie d'un lien avec l'au-delà » aurait été réduite à « confiance : modalité chaleureuse et sympathique de la relation intersubjective ». "Car cette traduction, unanimement saluée comme "hérétique" ou "dérangeante", ne prend des libertés avec le Dogme que pour noyer le message divin dans l'immanence démocratique " (p. 221). Brillant mais rapide, l'acte d'accusation en reste en fait à quelques expressions glanées dans l'Évangile de Matthieu. Celles-ci semblent suffire à l'auteur pour déplorer plus largement la fin de toute pensée de la transcendance au moment où «l'humanité n'est éblouie que par elle-même ».

23. Northrop FRYe, Le Grand Code, La Bible et la littérature (The Great Code, 1981), Paris, Seuil, coll. " Poétique ", 1984. Classique de la critique littéraire de la Bible.

24. Sur cette dynamique: Pierre-Maurice Bogaert, op. cit.

25. Alain FinkielkRaut, "Les Écritures de l'Humanité-Dieu », in Alain FINkIELKRaUt, L’imparfait du présent, Paris, Gallimard, 2002, p. 218-221. 
Des comminations côté catholique aux imprécations côté laïque, en passant par le silence parlant de certains cercles littéraires, la violence symbolique n'a donc pas manqué à la réception. Il n'en a pas fallu plus pour que Boyer reprenne aussitôt la plume pour défendre l'œuvre de traduction contre les nostalgiques de la tradition. Dans La Bible, notre exil, il montre comment les catholiques intransigeants et les gardiens laïques des lois de la transcendance se rejoignent pour « réduire l'événement poétique, linguistique et religieux de la Bible à une mémoire scolaire de dictons, d'expressions figées» $\left(^{\text {p. 99 }}{ }^{26}\right.$. Il prend ainsi à témoin le «millefeuille sémantique et linguistique " des innombrables traductions dont les sources se perdent dans la nuit des temps et dont les interprétations sont infinies, pour plaider la cause d'une éthique vivante de l'héritage culturel. Non pas repli frileux sur un langage possessif mais accueil constant de l'autre au bout de l'épreuve de la perte. Ouverture au monde qui est le propre d'un christianisme qui «n'a que la langue de l'autre pour se dire à lui-même en se disant aux autres » (p. 121). De Paul qui ne peut délivrer autrement son message que «dans la faiblesse de la chair " $(\mathrm{Rm} 6,19)$ à Dei Verbum qui reconnaît l'incarnation du Verbe dans le corps faillible de la lettre, les références doctrinales ne manquent pas. Au schéma légaliste de Finkielkraut qui oppose la transcendance religieuse à l'immanence démocratique, Boyer peut ainsi répondre que la « transcendance gît dans la poussière des mots que nous employons familièrement, fait effraction jusque dans le ridicule et la pauvreté de nos langages » (p. 109). L'essai tente finalement une explication de cette «culture patrimoniale » qui, de droite comme de gauche, attaque la «culture de la traduction »: ce serait précisément la «société de la vitesse, de la dispersion et du virtuel » qui exacerberait les replis conservateurs (p.113). Peu après la parution de l'essai, Finkielkraut invita loyalement Boyer pour en débattre dans l'émission Répliques qu'il anime sur France-Culture $(14 / 09 / 02)^{27}$. Au cours du débat, au demeurant fort cordial, Boyer semble avoir eu quelque difficulté à convaincre ses interlocuteurs de dépasser le seul plan de l'énumération plus ou moins scandalisée des mots "déplacés " par la nouvelle traduction. Confirmation partielle de sa thèse sur la société de vitesse qui évite la complexité des choses?

En tout état de cause, et comme pour vérifier le vieil adage selon lequel toute bonne histoire finit toujours par une chanson, peu après ce dialogue de sourds, la presse plébiscite le Cantique des cantiques, produit dérivé de la BNT (traduction Berder/Cadiot), mis en musique par le chanteur Alain Bashung. "Alien Bashung, Païen ou liturgique, deux albums où il "déchante" ", c'est par ce titre enjoué que Libération (18/10/02) annonce la sortie prochaine de deux CD de « la force tranquille du rock français » : "L'imprudence », recueil de morceaux récitatifs

26. Frédéric BOYER, La Bible, notre exil, Paris, POL, 2002.

27. Boyer se vit à l'occasion opposé à Gérard Leclerc, éditorialiste à la France Catholique (hebdomadaire dans la mouvance intransigeante) et collaborateur au Figaro, qui venait de publier Les Dossiers brûlants de l'Église (Paris, Presses de la Renaissance, 2002), vigoureuse défense et illustration du rigorisme pontifical. 
qui s'éloignent du rock ; "Cantique des cantiques », duo psalmodié entre Bashung et Chloé Mons, sa nouvelle épouse, sur fond instrumental de Rodolphe Burger. Ce second album fut enregistré lors de la cérémonie de mariage du chanteur et de la comédienne dans une petite église du Pas-de-Calais à l'été 2001. "Pétri de doutes et d'(in)croyances, le chanteur, qui n'est pas à une contradiction près, passe ainsi du païen au sacré, et vice versa » (Ludovic Perrin). On apprend dans Le Nouvel Observateur («Disques : la bande à Cadiot», 28/11/02) que c'est Alferi qui a présenté Cadiot à Burger (rocker du groupe Kat Onoma, mais aussi philosophe-musicien vedette de Vacarme, ami de Jacques Derrida, le père d'Alferi), lequel a présenté Cadiot à Bashung. Un nouvel éditeur de disques voit ainsi le jour, "La dernière bande ", avec le Cantique comme premier produit auquel le groupe Bayard s'est associé. Affinités électives qui montrent toute la plasticité sociale de notre configuration biblique qui se déplace ici vers quelques quartiers branchés de l'industrie du spectacle.

À l'approche des cadeaux de fin d'année, les presses catholique et musicale saluent de concert "Les sacrés mots d'amour d'Alain Bashung (La Croix, 2/12/02) : "Le plus vieux chant du monde se révèle l'événement musical de l'année » (La Vie, 5/12/02) ; "Depuis longtemps la voix du Cantique n'avait pas ainsi retenti sur la place publique » (Croire aujourd'bui, 1/12/02) ; "Élégant, riche, pur, ce dialogue amoureux est d'une douce splendeur " (Le Monde de la musique); "Biblique mais charnel, le texte gagne encore en sensualité dans l'entrelacement hypnotique des voix visiblement aimantes, lovées sur une musique minimaliste, répétitive »(Les Inrockuptibles). Mais la surprise de fin d'année provient surtout du Figaro et de Famille chrétienne qui, un an auparavant, tiraient à boulets rouges sur la «Bible Bayard». Le premier parle de «beau mariage ": "la réverbération naturelle de l'église, l'élégance de la musique, l'alternance de lyrisme oriental et de simplicité quotidienne dans le texte: un court disque inondé de lumière et de paix, sans que se déchire le beau voile des mystères amoureux »(Le Figaro, 21/10/02). Le second trouve dans ce «Bashung inattendu » une «belle façon de revisiter ce magnifique texte biblique » (Famille chrétienne, 11-17/01/03). La guerre des Bibles serait-elle terminée, tout bonnement évaporée dans un disque de fête?

Sans doute non, à en croire Mgr Jean-Pierre Cattenoz, nouvel évêque d'Avignon, qui, lors d'une homélie prononcée en mai 2003, rejoint l'évêque de Saint-Dié (Mgr P.-M. Guillaume dans le numéro de Képhas évoqué) pour condamner publiquement, sans la nommer, « une Bible qui semble avoir gommé volontairement tout le vocabulaire que l'Église avait forgé tout au long de son histoire pour rendre compte de la richesse du Mystère [...]. Non, cette Bible n'est pas celle de l'Église !». Le prélat mêle à sa monition une autre publication, apparemment plus scandaleuse, celle d'un livre intitulé L'Église et l'art d'avantgarde. De la provocation au dialogue (Albin Michel, 2002) ${ }^{28}$. Il n'en fallut pas

28. Ouvrage illustré, doublement écrit par Gilbert Brownstone, expert international en art contemporain, et Mgr Albert Rouet, évêque de Poitiers. À l'initiative du groupe de travail Arts- 
plus au quotidien d'extrême droite Présent (11/07/03) pour relancer, sous la plume coriace de Madiran, l'idée de soumettre la «Bible cracra-badaboum et le livre catho-porno » à l'appréciation de la Congrégation romaine pour la doctrine de la foi.

On peut douter de l'accueil d'une telle proposition dans une Église qui, à l'instar de l'avis nuancé et dilatoire de la commission épiscopale sur l'imprimatur, semble aujourd'hui préférer l'équilibre des tensions au conflit doctrinal d'un autre âge. Du moins pour tout ce qui relève des questions d'esthétique ou de culture, là où la morale de l'« ordre naturel » n'est pas directement en cause. Au cours de l'année 2002, cette même commission a dû d'ailleurs condamner les fameuses "clefs de lecture " de l'édition 2001 de la Bible de Jérusalem, sous la pression de l'association pour l'Amitié judéo-chrétienne, présidée par Paul Thibaud, ancien directeur de la revue Esprit (de 1977 à 1988), et du Sidic ${ }^{29}$. Ces « clefs» tendent à christianiser abusivement le Premier Testament et à nier l'identité juive des Écritures, alors même que, dans la suite de la déclaration conciliaire Nostra aetate (1965) sur les relations entre l'Église et les religions non chrétiennes, la Commission biblique pontificale vient de publier un document, Le peuple juif et ses saintes Écritures dans la Bible chrétienne (2001), qui remet en cause toute annexion chrétienne de l'héritage judaïque et reconnaît pleinement la lecture juive des Écritures, en parallèle fécond et dialogique avec la tradition chrétienne.

\section{Enjeux et publics}

Entre prudence épiscopale, rejets passéistes, consensus musical, emballement médiatique ou mépris littéraire, la réception de l'œuvre révèle ses champs imbriqués et ses publics mobiles. Les uns circonscrits à des chapelles, les autres ouverts aux flux mêlés des Fnac. Aussitôt capté par les médias, le best-seller se forge au fil d'un commentaire qui s'en tient surtout aux petites curiosités de vocabulaire et met en scène les vedettes littéraires engagées dans l'aventure. La fausse ambivalence entre texte sacré et traduction profane, habilement entretenue par les journalistes français, constitue pour eux une ressource narrative précieuse, par les effets d'ironie et de surprise qu'elle engendre auprès d'un lectorat inégalement instruit du statut et de la portée du texte biblique. Le relevé amusé des audaces d'une traduction qui a largué les amarres avec la Vulgate procède rarement d'une critique sur le fond. Ni opposition ni adhésion à ce qui pourrait être

Cultures-Foi mis en place en 1997 par l'épiscopat français, ce livre réunit et commente des œuvres représentant le corps humain aujourd'hui (de Francis Bacon à Lee Wagstaff en passant par Gilbert \& George). Pour l'évêque d'Avignon, les images de chair offerte, mutilée ou disséquée «ne sont que le reflet des pulsions morbides et sexuelles qui habitent le cour de l'homme blessé et défiguré par le péché et ne sauraient (me) conduire à percevoir la transcendance du Beau ".

29. Service information-documentation juifs-chrétiens, organisme catholique animé par des religieuses de la congrégation Notre Dame de Sion. 
pris, aux yeux des nostalgiques de la croisade, pour une contestation radicale de l'universalité et de l'unicité de l'héritage biblique. Dans un marché de l'information à flux tendus, mieux vaut miser sur les jeux de l'étrange et du familier que d'ennuyer les lecteurs à en chercher les raisons. L'impression de superficialité que dégage la lecture de la presse pourrait éventuellement s'expliquer comme un nouvel effet de la sécularisation ou bien, de façon plus circonstancielle, par le faible niveau de culture biblique des journalistes français comparé à celui des voisins francophones. Mais ici comme ailleurs, les limites de la critique procèdent surtout de l'art d'informer en attirant l'attention du chaland multiple dans une société plurielle. Savant équilibre entre plus petit dénominateur sémantique (les mots plutôt que le style de la traduction) et lignes de fuite philosophiques (ambivalence entretenue sur le statut symbolique de la Bible aujourd'hui).

Hormis les attaques intransigeantes qui campent dans leur marginalité active, la fausse querelle de clocher entre libéraux ( "Une Bible qui réveille la lecture ", "Le christianisme n'est pas une religion du livre ", "La traduction qui ouvre le chemin ») et conservateurs ( Une Bible intrinsèquement rebelle », « opération commerciale pour l'intelligentsia branchée ») cache mal l'épuisement historique d'un conflit symbolique. Partenaire obligé de la "Bible des écrivains ", l’Église catholique n'en a que plus d'embarras à prendre position. Valider la traduction profane (i.e. scientifico-littéraire) d'un texte reçu comme sacré dans une religion qui prône pourtant l'accessibilité universelle de son message ne va pas de soi. Les tensions qui animent l'Église et les réseaux divers qui la traversent laissent, malgré tout, entrevoir les lignes de forces et de rupture qui tissent la trame sociologique de la traduction. Ainsi, la tentative apparemment réussie du groupe Bayard de prendre place sur le marché du livre biblique et la résistance que le Cerf, allié pour la circonstance au groupe Média-Participations (via FleurusMame), lui a opposé, au prix de la condamnation épiscopale de ses propres «clefs de lecture " pour défaut de «discernement ecclésial ». Retournement étonnant de situation, où une réédition hâtive de la Bible de Jérusalem, propriété du Cerf qui avait initié cette traduction révolutionnaire ou «dominicaine » longtemps combattue par les intransigeants, se voit aujourd'hui soupçonnée de déviance antisémite. Dans cette concurrence éditoriale à somme non nulle, le rôle dévolu à certaines signatures liées à tel ordre religieux n'est pas négligeable : aux jésuites la défense et l'illustration de la «traduction Bayard ", aux dominicains sa critique mesurée ou radicale ${ }^{30}$. Ces quelques mouvements de position ne peuvent se comprendre indépendamment des tensions plus générales entre le recentrement romain autour d'un catholicisme d'identité et une Église nationale encore traversée de courants intellectuels ou missionnaires qui réactivent la

30. Le bimensuel jésuite Croire aujourd'hui (octobre 2001) s'est fait ainsi un solide défenseur de la BNT, sous les plumes expertes de Pierre Gauffriau et de Pierre GIBERT. Côté dominicain, on relève notamment l'analyse nuancée de Jean-Michel MALDAMÉ (Esprit et Vie, décembre 2001) et les critiques plus radicales des frères Venard et Sed déjà signalées. 
logique sécularisatrice du christianisme ${ }^{31}$. Il reste que le débat autour de la BNT a principalement mobilisé le monde catholique, l'expertise des protestants libéraux ayant discrètement validé l'expérience ${ }^{32}$. Malgré la participation notable mais isolée du jeune rabbin Marc-Alain Ouaknin (traduction du livre de Jonas), les membres de la communauté juive se sont faits encore plus discrets. Sans doute parce que la traduction biblique en langues modernes est une tradition typiquement chrétienne alors que la Torah n'est, au mieux, traduisible qu'à la marge de la lecture infinie qu'elle engendre et qui, Talmud aidant, structure la communauté ${ }^{33}$.

Hors des confessions, le destin littéraire de notre traduction n'a jusqu'alors que peu dépassé le stade de la critique journalistique. L'abondance et la variété de notre dossier de presse attestent la notoriété laïque de l'ouvrage. Son bon chiffre de ventes aussi. Mais la rencontre du succès médiatique et commercial laisse encore ouverte la question de la portée littéraire de l'œuvre de traduction. L'《énergétique de la traduction » développée par Boyer, Cadiot et Alferi dans Vacarme coexiste ainsi sans dialogue possible avec la "politique du rythme " réaffirmée par Meschonnic dans L'Infini. Outre quelques essais malheureux avec divers auteurs sollicités par Boyer, le refus d'un écrivain reconnu de se lancer dans la traduction au prétexte que la Bible est un «mauvais livre » témoigne de la résistance du monde des lettres à une entreprise où la liberté de création est apparemment sujette à caution ${ }^{34}$. Où le principe de fidélité inhérent à la traduction biblique risque de faire de l'ombre au "régime de singularité » qui peut définir l'identité de l'écrivain ${ }^{35}$. On peut aisément comprendre que, suivant le profil des auteurs, participer à cette traduction revête des significations différentes. Pour les écrivains consacrés par la critique littéraire, l'aventure fait preuve d'audace et de liberté; pour les plus médiatisés, elle conforte une image de croyant ou à l'inverse d'iconoclaste; pour les moins connus, elle constitue une épreuve qualifiante. Côté biblistes, peu mis en valeur par la presse, l'épreuve de la traduction n'a pas moins contribué à la reconnaissance publique d'un savoir qui se détache d'une longue tutelle ecclésiale ${ }^{36}$.

31. Sur l'évolution de ces tensions, voir notamment: Jean BaUdounn, Philippe PorTIER, dirs, Le mouvement catholique français à l'épreuve de la pluralité, Rennes, Presses universitaires de Rennes, 2002.

32. Dans le dossier sur les «Bibles en traduction » du Cahier biblique Foi et vie ( $\mathrm{n}^{\circ}$ 41, 2002), la BNT est saluée pour son travail de renouvellement littéraire malgré quelques défauts de concordance et diverses consonances malheureuses (D. LYS, "La Bible en quête de langage(s) ", p. 65-78).

33. Célèbres sentences talmudiques à propos de la traduction : "Il ment celui qui rend mot pour mot; il blasphème celui qui y ajoute quelque chose "; "Les choses écrites, tu ne peux les transmettre oralement ; les choses orales, tu ne peux les transmettre par écrit ». Débat constitutif qui n'exclut pas l'histoire diasporique de la traduction juive, de la célèbre Septante grecque à la Bible française du rabbinat aujourd'hui.

34. Il s'agit d'Emmanuel Hocquard dont les principes de traduction collective et à haute voix ont été repris par les poètes de la BNT, notamment Roubaud.

35. Nathalie HeINICH, Etre écrivain, Création et identité, Paris, La Découverte, 2000.

36. Conjectures développées dans notre enquête directe auprès des traducteurs. 
Il manque sans doute à notre dossier de réception un espace de réflexion sur les enjeux culturels de la traduction d'un texte, certes "grand code de l'art » occidental, mais dont l'exégèse relève encore, pour l'essentiel, des instituts de théologie. Le seul débat d'idées marquant l'épreuve publique de l'ouvrage a en effet opposé les tenants de la culture patrimoniale (Finkielkraut rejoignant pour une part les objections des catholiques identitaires) à ceux d'une culture de la traduction (Boyer principalement, avec La Bible, notre exil). Traduction que Paul Ricœur érige en principe "d'équivalence sans identité » aux fins de servir le «projet d'une humanité sans briser la pluralité initiale» (Le Monde, 25/05/ $2004)^{37}$. Le Cantique remixé par Bashung et qui fait momentanément consensus rappelle que cet équilibre entre équivalence et altérité reste fragile.

Dans ce qu'il dit comme dans ce qu'il tait, notre dossier de presse ne nous éloigne pas seulement d'une confrontation abstraite entre médias et religions. Les tensions qu'il met en scène entre avant-garde littéraire, autorités ecclésiales, exégèse scientifique, industrie du livre, courants conservateurs et libéraux, traduisent précisément une série d'ambivalences sans lesquelles la BNT n'aurait sans doute pas fait événement : projet fluctuant entre expérimentation poétique et renouvellement missionnaire; produit naviguant entre cercle lettré et grand public; réception oscillant entre registre profane et sacré. Ce que l'historien pourrait présenter comme la rencontre, longtemps différée en France, entre la déconfessionnalisation du texte biblique, le génie de la langue française, le développement des sciences humaines et le goût public du retour aux sources, trouve dans ce dossier de presse l'illustration vivante des formes à la fois conflictuelles et ludiques de reclassement culturel d'un héritage canonique. Les effets de composition entre logiques plurielles que nous avons analysés mettent en tout cas l'accent sur l'efficacité symbolique des équivoques. À la détraditionalisation, au présentisme et à la réflexivité comme traits culturels de la modernité avancée, faudrait-il sans doute ajouter ici le malentendu productif ${ }^{38}$.

Pierre LASSAVE Centre d'Études Interdisciplinaires des Faits Religieux

37. Divers textes du philosophe sur la traduction ont été précisément réunis par l'éditeur de la BNT (Paul RICCEUR, Sur la traduction, Paris, Bayard, 2003).

38. Sur ce thème: Franco LA CECLA, Le malentendu (Il malintenso, 1997), trad. de Annemarie Sauzeau, préf. de Marc Augé, Paris, Balland, coll. «Voix et regards », 2002. 


\section{Résumé}

Au début du troisième millénaire, une nouvelle traduction française de la Bible, fondée sur l'exégèse critique et réécrite par des écrivains contemporains de renom, a connu le destin d'un best-seller. Son volumineux dossier de presse révèle les équivoques productives d'une entreprise qui tient à la fois du renouvellement missionnaire, de l'expérimentation littéraire et de l'opération éditoriale. La focalisation ludique sur le déplacement des mots de la tradition occupe le devant de la scène médiatique et fait de cette traduction un événement culturel.

Mots clés: Bible, média, traduction biblique, bible et littérature, exégèse.

\section{Abstract}

Right at the start of the third millenary, a new French translation of the Bible, based on critical exegesis and rewritten by famous authors, has grown into a best seller. Its large press-book shows the productive ambiguity of an enterprise that is at once missionary, literary and publishing operation. Media jokes on strange change of traditional words cause a cultural stir.

Key words: Bible, mass-media, biblical translation, Bible and literature, exegesis.

\section{Resumen}

Al principio del tercer milenario, una nueva traducción francesa de la Biblia, basada en la exégesis crítica y reescrita por escritores contemporáneos famosos, ha conocido el destino de un best-seller. Su espesa revista de prensa revela los equivocos productivos de una empresa que se debe a la vez de la renovación misionaria, de la experimentación literaria y de la operación editorial. La focalización lúdica sobre el desplazamiento de los términos de la tradición ocupa el primer puesto mediático y hace de esta traducción un acontecimiento cultural.

Palabra clave: Biblia, médias, traducción biblica, Biblia y literatura, exégesis. 